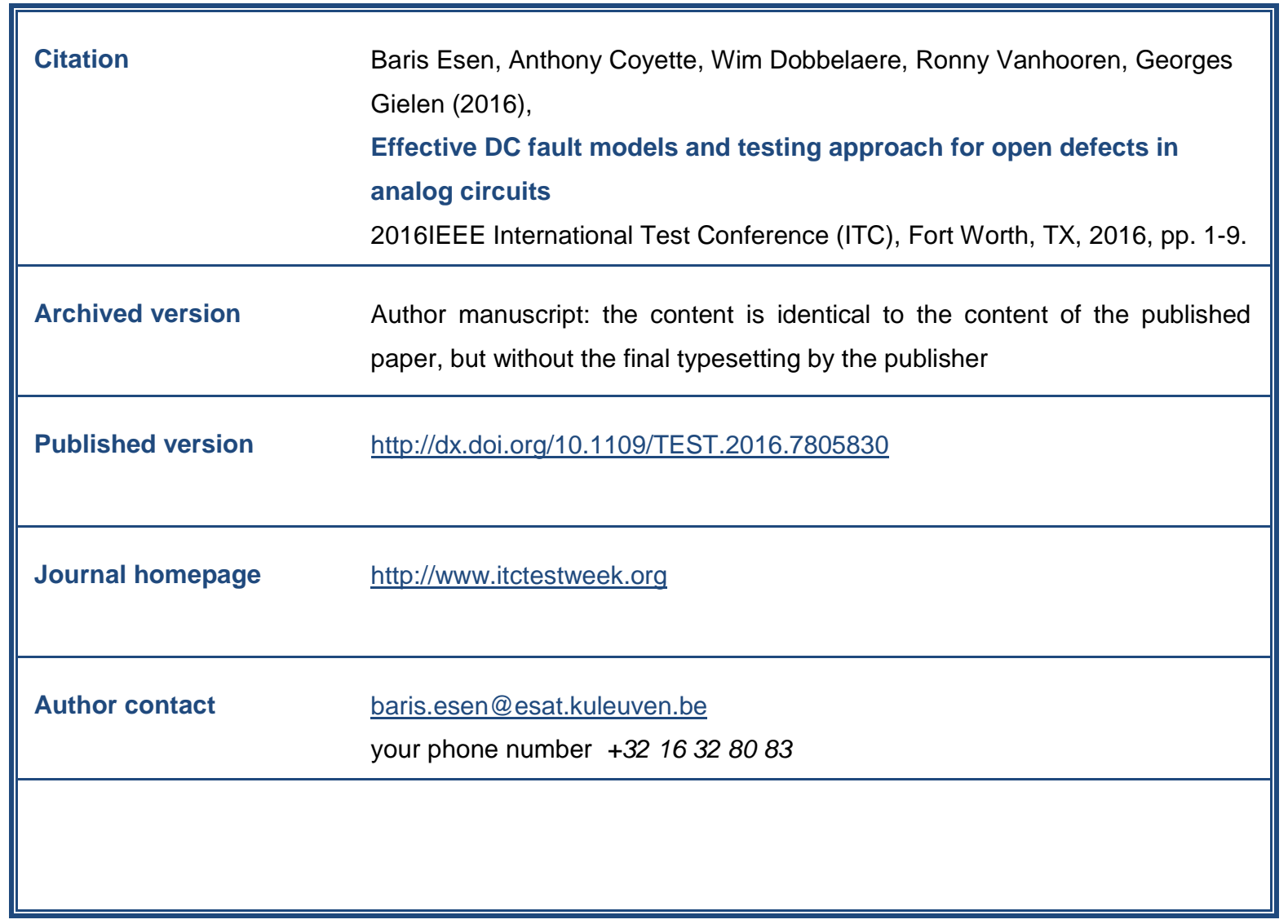

(article begins on next page) 


\section{Effective DC Fault Models and Testing Approach for Open Defects in Analog Circuits}

\author{
Baris Esen, Anthony Coyette \\ and Georges Gielen \\ Department of Electrical Engineering (ESAT) \\ KU Leuven \\ Leuven, Belgium
}

\author{
Wim Dobbelaere and Ronny Vanhooren \\ APG Automotive Mixed Signal \\ ON Semiconductor Belgium \\ Oudenaarde, Belgium
}

\begin{abstract}
The detection level of defects in today's mixedsignal ICs lags behind the extremely high demand of industries such as automotive. This is mainly because analog blocks in these ICs have high test escape rates as a result of the typical testing based on the performance specifications. Defect-oriented techniques have been proposed to solve the problem of this poor fault coverage for analog circuits. Their effectiveness in practice is however still limited due to the inadequate fault models used to represent physical failures. This paper presents a new open-gate DC fault model. Experimental results on fabricated test circuits in $0.35 \mu \mathrm{m}$ BCD technology are used to validate the proposed fault model and the commonly used high-value-resistance model. Finally, a new testing approach to detect the corresponding open defects in analog circuits is discussed, which is based on forcing the transistors outside their designed operation region.
\end{abstract}

Keywords-mixed-signal test; defect-oriented test; fault modeling; open defects; open-gate defect

\section{INTRODUCTION}

The field of IC testing plays the role of filter between the non-perfect manufacturing in practice of silicon dies in foundries and the market requiring fully functional electronic devices. Today's semiconductor process technologies inevitably result in the fabrication of chips including defects, which have to be detected during testing. At the same time, industries like the automotive one are demanding absolute reliability, while facing the emergence of application systems (i.e. vehicles) involving a continuously increasing number of chips. As a result, they have to cope with the multiplication of the failure probabilities. Hence, the rising demand for below part per million (sub-ppm) defect-level ICs in these industries [1].

While the digital core generally constitutes the major part of a typical mixed-signal IC, defect levels that are significantly below ppm have been achieved as a result of the structured test approach using well-defined fault models (e.g. stuck-at, bridging, delay) [2]. Therefore, the dominant portion of test escapes is still caused by the defects occurring in the analog circuitry. The reason is that the analog blocks traditionally are tested in an empirical way in accordance with the functional and performance specifications. Typically, well-known performance characteristics for each analog block are individually measured, and the chips with values that are outside the predefined specification limits are rejected as defective ICs. These measurements vary from large numbers of simple DC characterizations to complex high-frequency counterparts, which usually require very expensive test equipment to perform. As a result, the specification-based analog tests are long and expensive. Furthermore, there is no quantification of the actual coverage of real physical defects by the applied specification tests. This lack of defect-coverage quantification often results in poor fault coverage and unacceptably high test escape rates [3].

In order to improve the analog fault coverage and to decrease the analog test cost, defect-oriented testing methods have been proposed [4]-[7]. In these methods, possible defects that might occur in an analog circuit are modeled and simulated such that a set of tests can be generated to detect these defects. In contrast to specification-based methods, these tests can be selected to be simple and of relatively low cost. In addition, the actual fault coverage for every IC can be foreseen accurately, which further enables improvements in case of undesiredly high test escape rates. The success of defect-oriented testing, however, strongly relies on the fault models that are used to simulate the physical fabrication abnormalities.

Two types of fault models have commonly been used in analog defect-oriented testing: 1) parametric fault models, which represent the non-nominal behavior due to the variability inherent to the VLSI manufacturing process, and 2) the catastrophic fault models, which represent a change in the topology due to a physical defect; for instance, caused by a dust particle or missing material in the process. Parametric fault models have been well defined as random variations and have successfully been used not only in testing, but also in process-invariant design techniques [8]-[10]. On the other hand, there are no commonly accepted catastrophic fault models, and especially not for open defects [11]. While the imperfections that are causing short circuits are fairly adequately represented by small resistances added to the original circuitry, the large-resistance counterparts for modeling open defects are proven to be not generally correct [12]. Furthermore, this resistive model is not even effective when it is used for the specific case of an open gate in DC simulations.

In this paper, open defects in analog circuits are analyzed, and a new open-gate model for DC simulations is proposed. In Section II, the theoretical analysis of open defects is given with the proposed simulation fault models. In Section III, 


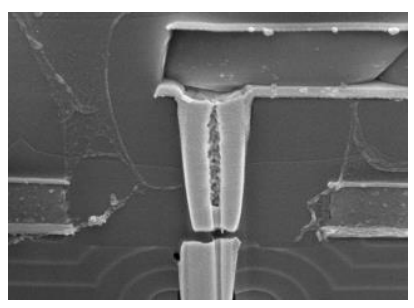

(a)

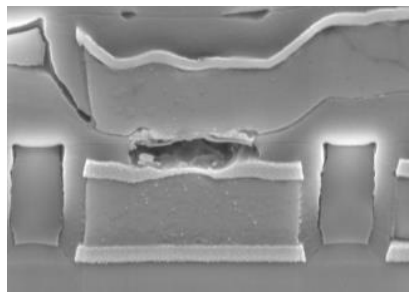

(c)

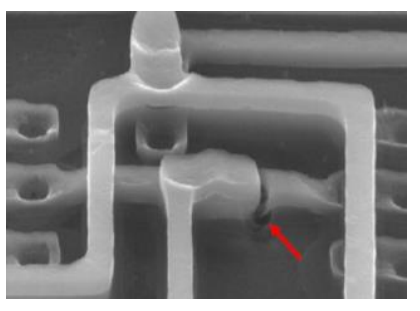

(b)

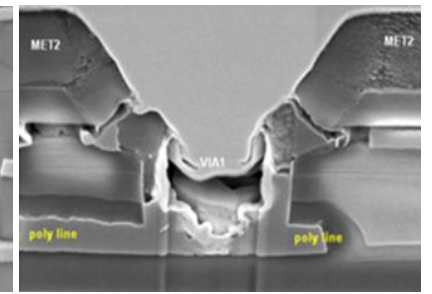

(d)
Fig. 1. Examples of open defects in a state-of-the-art semiconductor process: (a) open contact due to missing metal, (b) open metal due to overetch, (c) open contact due to broken metal, and (d) open contact due to micro cracks in metal.

experimental results are compared to the fault simulations which employ the proposed models. Finally, in Section IV, a new effective testing approach for open defects is discussed in comparison with the traditional tests using the experimental results. Conclusions are drawn in Section V.

\section{OPEn DEFECTS IN ANALOG CiRCUITS}

Physically, open defects are caused by missing or broken material in a semiconductor process, as illustrated by the examples in Fig 1. Two different types of effects can be observed as a result of these defects: floating gate terminals for one or more transistors and two or more floating analog branches. Since these cases are fundamentally different, the distinction in terms of theoretical analysis and fault modeling is also adopted in this paper. First, a detailed electrical analysis of open-gate defects is given, and a new fault model is proposed. Then, the open defects affecting the source or drain terminals are analyzed.

\section{A. Open-Gate Defects}

Open-gate defects occur when the gate terminal of a transistor is left disconnected from the controlling signal coming from the rest of the circuitry, generally, because of missing metal to poly contacts or a break in one of the metal traces. The other physical source, i.e. a break in polysilicon, is less likely to create an entire transistor with a floating gate in analog circuits due to the reasons that will be discussed later. Nevertheless, it is covered in this analysis for the sake of completeness.

During the emergence of defect-oriented test methods, it has been recognized that open-gate defects cannot be modeled by simple stuck-at faults in digital circuits or by high-value resistance model in analog domain. Therefore, initial work has concentrated on the detailed theoretical analysis of these defects. Complex capacitive models, which take the parasitic capacitances of the related transistor into account, have been proposed as a result of this analysis [12]. However, a common drawback shared by these models is that the operation-region dependence of the MOSFET capacitances is neglected not to further complicate the necessary calculations. This assumption is approximately correct for small-area transistors since the change in capacitance values is relatively small. However, it is, obviously, a limitation for larger transistors, which are usually employed by analog circuits with the goals of better matching between a pair of transistors or having a larger intrinsic gain from a single transistor, etc. Another limitation to these models is that the values for the other parasitic capacitances, such as the metal to gate overlap capacitance and the poly to bulk overlap capacitance, have to be assumed random in order to keep the models comprehensive. In the case of analog circuits, however, important assumptions about the relative size of these capacitances can be made to simplify the analysis. These assumptions will be introduced later in this section. Furthermore, injecting the parasitic capacitances in the fault simulations is redundant for most of the available simulators, since these parasitic effects are already taken into consideration by the transistor models.

Other works have focused on the testability of open-gate defects by categorizing the behavior in terms of defect parameters including the parasitic capacitances mentioned before. Detectable regions for static voltage testing and dynamic voltage testing have been analyzed at the digital gate level with the use of capacitive models [13]. The application of $\mathrm{I}_{\mathrm{DDQ}}$ testing has also been examined to find guaranteed detection regions where a significant quiescent current flows when an open-gate defect occurs in digital circuits [14]. An extension of this approach to analog circuits has also been proposed, where it has been proven that stuck-open or stuck-off models are not effectively representing the open-gate defects in analog circuits [15]. Moreover, a unified approach has been presented to generate a set of tests, which assures the detection of an open-gate defect in the case of all the possible defect parameters that are unpredictable [16]. This method eliminates the need to calculate the layout and defect-location dependent parameters in the test generation procedure. However, this approach cannot provide any insight for the defects, since it is not possible to simulate the actual defective behavior. This lack of insight can cause redundancy in the selection of tests, which increases the overall test cost.

Floating-gate devices have been analyzed in previous works that are involving nonvolatile memory applications (e.g. EPROM, EEPROM, and Flash) [17]-[19]. The DC behavior of the floating-gate transistors in these memory cells has been modeled by a voltage-dependent voltage source to solve the capacitor-caused convergence problems in circuit simulators [20]. Dummy transistors have been used in the procedure of floating-gate voltage calculation in order to cover all the possible operation regions defined by the transistor models [21]. These approaches, however, have limited applicability to defective analog circuits, since all the parameters determining the induced gate voltage can be extracted from the layout.

In order to study the electrical structure of an open-gate defect, the analysis in this paper concentrates on n-channel 


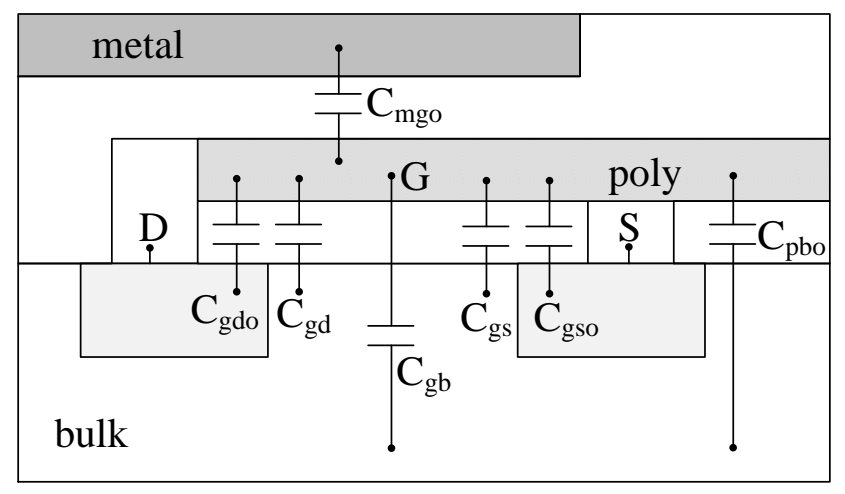

(a)

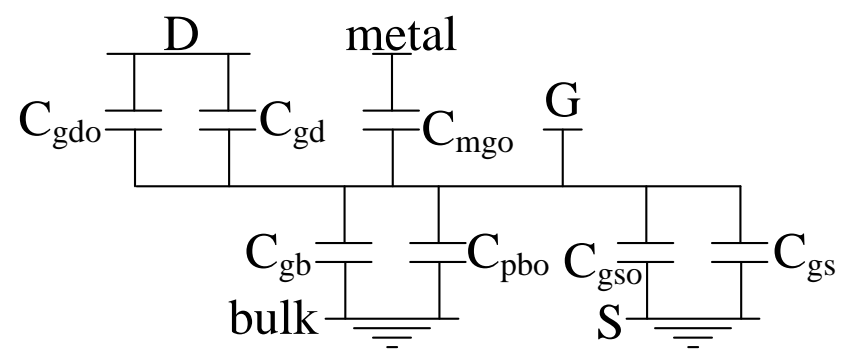

(b)

Fig. 2. (a) Crosssection of an n-channel MOS transistor and parasitic capacitances affecting the induced gate voltage. (b) Simplified electrical equivalent circuit.

MOS transistors for the sake of simplicity, though the same results can be obtained for $\mathrm{p}$-channel transistors, since the operation of $\mathrm{n}$-channel and $\mathrm{p}$-channel transistors are behaviorally similar. The conclusions made for $\mathrm{n}$-channel transistors, can easily be extended to p-channel transistors. When the gate terminal of a transistor is not connected resistively to any of the controlling signals, the induced gate voltage is determined by the effect of the capacitive couplings with the neighboring nodes. These capacitances to the related neighboring nodes are demonstrated in Fig. 2(a). Some of these nodes are evidently the other terminals of the transistor under consideration, i.e. the source, drain and bulk terminals. The other node is the metal trace, which carries the controlling signal. Although the capacitance between the controlling signal and the floating gate is demonstrated as an overlap capacitance between metal and polysilicon, $\mathrm{C}_{\mathrm{mgo}}$, in Fig. 2(a), in general, it can be a capacitance between two metal layers or two polysilicon traces. The type and the value of this capacitance depend on the defect location, and both are considered to be random in previous work. The other overlap capacitances are the gate-to-source $\mathrm{C}_{\mathrm{gso}}$, the gate-to-drain $\mathrm{C}_{\mathrm{gdo}}$ and the polysilicon-to-bulk $\mathrm{C}_{\mathrm{pbo}}$ overlap capacitances where the value of the latter depends on how the transistor gates are routed in the layout. Finally, there are also the gate to source, drain and bulk capacitances $\mathrm{C}_{\mathrm{gs}}, \mathrm{C}_{\mathrm{gd}}$, and $\mathrm{C}_{\mathrm{gb}}$, which are strongly dependent on the operation region of the transistor.

The electrical equivalent of this capacitive network is shown in Fig. 2(b), where it is assumed that the source and the bulk terminals of the n-channel transistor are both connected to ground in order to simplify the analysis. The conclusions and

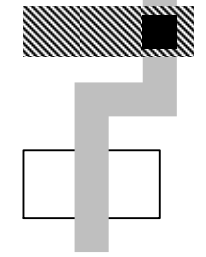

(a)

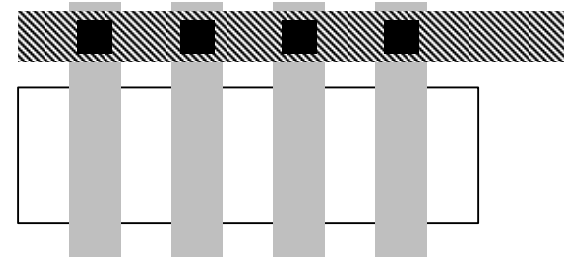

(b)
Fig. 3. Typical gate routing of transistors in (a) digital and (b) analog circuits.

the comparison with the experimental results, however, will be generalized later. From this equivalent network, the induced gate voltage can be written as:

$$
V_{g s}=\frac{\left(C_{g d}+C_{g d o}\right) V_{d s}+C_{m g o} V_{m s}+Q_{0}}{C_{g s}+C_{g s o}+C_{g d}+C_{g d o}+C_{g b}+C_{p b o}+C_{m g o}}
$$

where $\mathrm{Q}_{0}$ is the random initial charge trapped in the gate during the fabrication. The effect of this charge can be significant for small transistors, since it behaves as an offset voltage, which is inversely proportional to the total capacitance of the gate node. However, for larger transistor sizes, as is typically the case in analog circuits, it can safely be neglected, if the fabrication process does not include any abnormal charge insertion. This important assumption will be proven by the experimental results in Section IV.

The solution for (1) is not straightforward even with the above assumption, due to the aforementioned dependence of the parasitic capacitances on the operation region of the transistor, i.e. the value of $\mathrm{V}_{\mathrm{gs}}$ itself. Furthermore, both $\mathrm{C}_{\mathrm{mgo}}$ and $\mathrm{C}_{\mathrm{pbo}}$ are determined by the location of the defect. However, some useful assumptions can also be made for these capacitances, using the way analog transistors are routed in the layout. First of all, it is a common practice to divide analog transistors into equally sized fingers in the layout, as shown in Fig. 3. In addition, the routing of the gate signal is usually carried out by metal layers instead of using polysilicon, in contrast to digital transistors where it is more common to use polysilicon in the gate-level routing. Therefore, a break in the polysilicon or a single metal-to-poly contact missing cannot create an entire open-gate transistor in analog circuits. In other words, these two defect types can be represented by severe parametric faults rather than catastrophic faults. It might be observed that analog transistors with one or two fingers can create exceptions to this assumption. However, it will be proven by the experimental results in Section IV that the effect of the controlling signal is not significant in this specific case. The other possible reasons which can create open-gate transistors, are breaks in the metal tracks and missing contacts between metal layers where the overlap capacitances are negligible due to the small overlapping area.

Therefore, a simplified version of (1) can be written as:

$$
V_{g s} \cong \frac{\left(C_{g d}+C_{g d o}\right) V_{d s}}{C_{g s}+C_{g s o}+C_{g d}+C_{g d o}+C_{g b}}
$$


from which it is clear that $\mathrm{V}_{\mathrm{gs}}$ is a fraction of $\mathrm{V}_{\mathrm{ds}}$. Therefore, it can be concluded from (2) that an open-gate transistor cannot be in the linear region since $\mathrm{V}_{\mathrm{ds}}$ is always greater than $\mathrm{V}_{\mathrm{gs}}$. Furthermore, the following equations can be written for the other operation regions:

cut-off: $\left\{\begin{array}{l}V_{g s} \cong \frac{C_{g d o} V_{d s}}{C_{g s o}+C_{g d o}+W L C_{o x}} \\ V_{g s}<<\frac{V_{d s}}{2}\end{array}\right.$

subthreshold:

$$
\left\{\begin{array}{l}
V_{g s} \cong \frac{C_{g d o} V_{d s}}{C_{g s o}+C_{g d o}+C_{g b}} \\
V_{g s}<\frac{V_{d s}}{2}
\end{array}\right.
$$

saturation:

$$
\left\{\begin{array}{l}
V_{g s} \cong \frac{C_{g d o} V_{d s}}{C_{g s}+C_{g s o}+C_{g d o}} \\
V_{g s}<<\frac{V_{d s}}{2}
\end{array}\right.
$$

The first important conclusion which can be drawn from (3), is that open-gate transistors are in the cut-off mode only for very small $\mathrm{V}_{\mathrm{ds}}$ values. Another important conclusion from (4) is that open-gate transistors stay in the subthreshold mode as long as $\mathrm{V}_{\mathrm{ds}}$ is not greater than two times the threshold voltage. However, it can further be observed from (5) that $\mathrm{V}_{\mathrm{ds}}$ values slightly exceeding two times the threshold voltage are not enough for the transistor to enter the saturation region, since the ratio between $V_{g s}$ and $V_{d s}$ is significantly below one half in this region. This is mainly because $C_{g s}$ starts to increase as the induced $\mathrm{V}_{\mathrm{gs}}$ approaches the threshold voltage. Therefore, an open-gate transistor tends to stay in the subthreshold region for a large range of available $\mathrm{V}_{\mathrm{ds}}$ values. The minimum value of $\mathrm{V}_{\mathrm{ds}}$ ensuring the saturation region operation can be calculated in terms of the threshold voltage, $\mathrm{V}_{\mathrm{TH}}$, as:

$$
V_{d s, s a t}=\frac{C_{g s}+C_{g s o}+C_{g d o}}{C_{g d o}} V_{T H}
$$

Substituting the well-known values of $\mathrm{C}_{\mathrm{gs}}$ in the saturation mode and $\mathrm{C}_{\mathrm{gdo}}$ into (6) gives:

$$
V_{d s, s a t}=\left(2+\frac{2 W L C_{o x}}{3 W C_{o v}}\right) V_{T H}=\left(2+\frac{2 L C_{o x}}{3 C_{o v}}\right) V_{T H}
$$

where $\mathrm{C}_{\mathrm{ox}}$ is the oxide capacitance per unit area, $\mathrm{C}_{\mathrm{ov}}$ is the overlap capacitance per unit length, and $\mathrm{W}$ and $\mathrm{L}$ are the electrical width and length of the transistor. Equation (7)

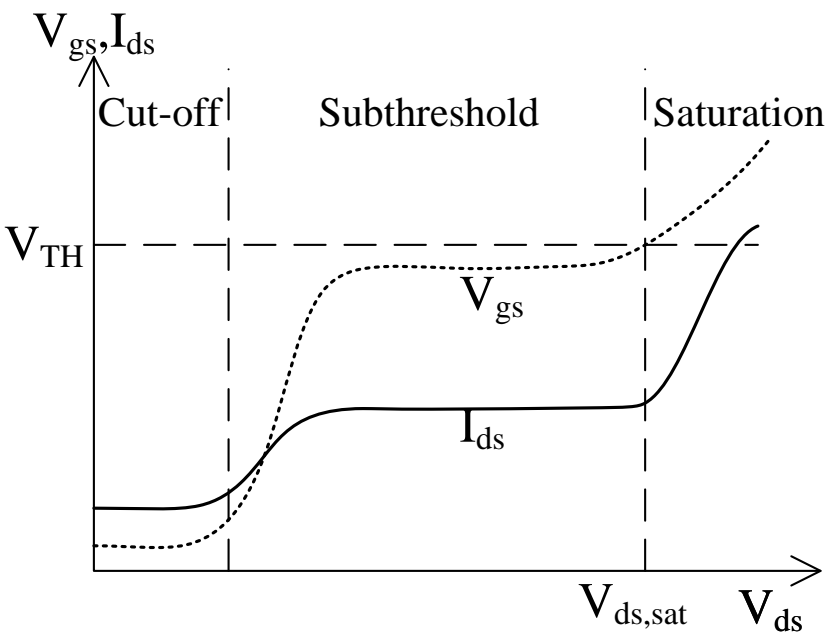

Fig. 4. Theoretical $V_{g s}$ and $I_{d s}$ versus $V_{d s}$ curve for an open-gate transistor.

suggests that $\mathrm{V}_{\mathrm{ds} \text {,sat }}$ is always greater than two times the threshold voltage and increases as the transistor length increases, since the second term in (7) is proportional to the transistor length. Furthermore, the second term in (7) can be calculated to be around 2 to 3 for a minimum-length transistor when using typical values of $\mathrm{C}_{\mathrm{ox}}$ and $\mathrm{C}_{\mathrm{ov}}$. Considering that the ratio between the available supply voltages and the threshold voltages decreases with every technology node, an open-gate transistor operates in the subthreshold region for a larger portion of the available $\mathrm{V}_{\mathrm{ds}}$ values with every new technology node. These conclusions are summarized in Fig. 4.

A more accurate analysis of the operation region dependence of the intrinsic capacitances can be made according to [22]. This would result in continuous transitions for the $\mathrm{C}_{\mathrm{gs}}$ and $\mathrm{C}_{\mathrm{gb}}$ capacitances where the sum of the two decreases from $\left(\mathrm{WLC}_{\mathrm{ox}}\right)$ to $\left(2 / 3 \mathrm{WLC}_{\mathrm{ox}}\right)$ as the $\mathrm{V}_{\mathrm{gs}}$ increases. It is important to note that the value of $\mathrm{V}_{\mathrm{ds} \text {,sat }}$ remains the same independent of this analysis. However, the ratio between $\mathrm{V}_{\mathrm{gs}}$ and $\mathrm{V}_{\mathrm{ds}}$ can be expressed with a higher accuracy in all the three possible regions as:

$$
\frac{C_{g d o}}{W L C_{o x}} \leq \frac{V_{g s}}{V_{d s}} \leq \frac{3 C_{g d o}}{2 W L C_{o x}}
$$

Thus, an open-gate transistor can be modeled in DC by a transistor having the same electrical properties and operating in the subthreshold region. In fault simulations, this is achieved by a voltage-dependent voltage source, which has a maximum amplitude smaller than the threshold voltage, and which is connected between the gate and the source terminals of the transistor. The voltage difference between the drain and the source terminals is used as controlling voltage in order to have induced gate voltage only when a voltage difference occurs between the drain and source terminals, as is analyzed. This model has significant advantages over the models proposed previously. First, it is not needed to calculate or duplicate the intrinsic capacitances of the transistor. It is possible to use standard circuit simulators and conventional transistor models 


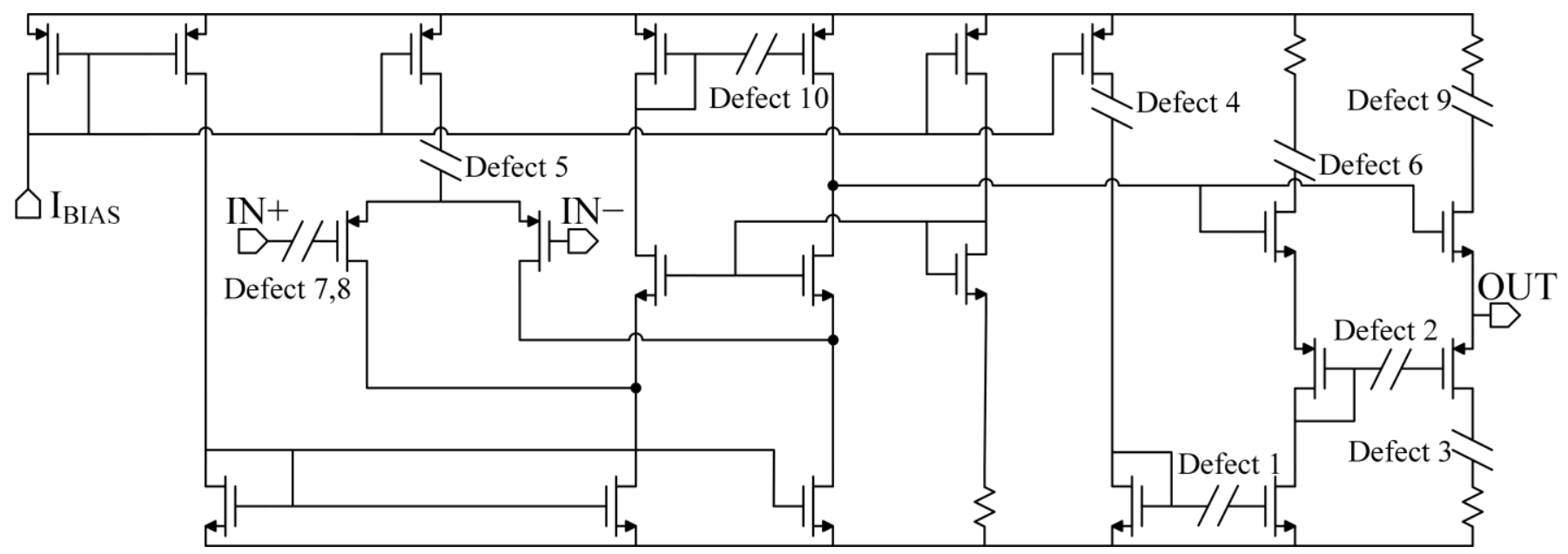

Fig. 5. Circuit diagram of the amplifier circuit and the injected open defect locations used in the experiments.

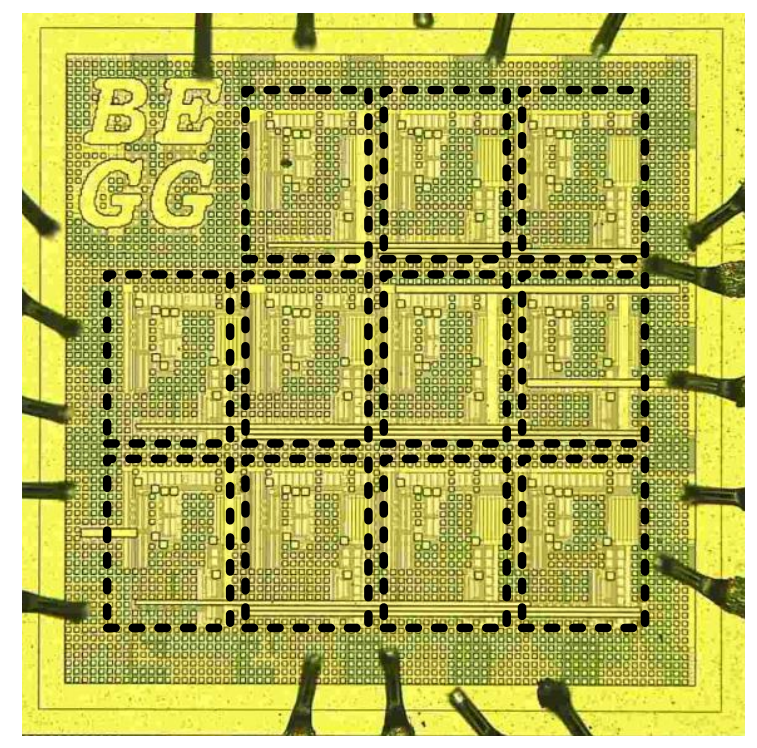

Fig. 6. Die micrograph of the produced test chip where each defectinduced amplifier circuit is highlighted, besides the one defect-free version.

without any extra effort. Finally, it is not required to estimate any defect parameters such as the location of the defect.

Furthermore, if needed, it is possible to use the proposed model to simulate the defective behavior more accurately. The ratio in (8) can be calculated according to the transistor length and the technology parameters to be used as the voltage gain of the voltage-dependent voltage source.

\section{B. Open-Drain/Source Defects}

Open-drain and open-source defects can be due to a break in metal tracks or missing contacts between two different metal layers. In this case, the gap between the two metals is filled with an oxide layer, which works as an insulator between the metal layers in normal conditions. As a result, the only available DC current on the affected branch is the combination of intrinsic leakage currents of the transistors and the tunneling current through the insulator. Capacitive coupling effects, in

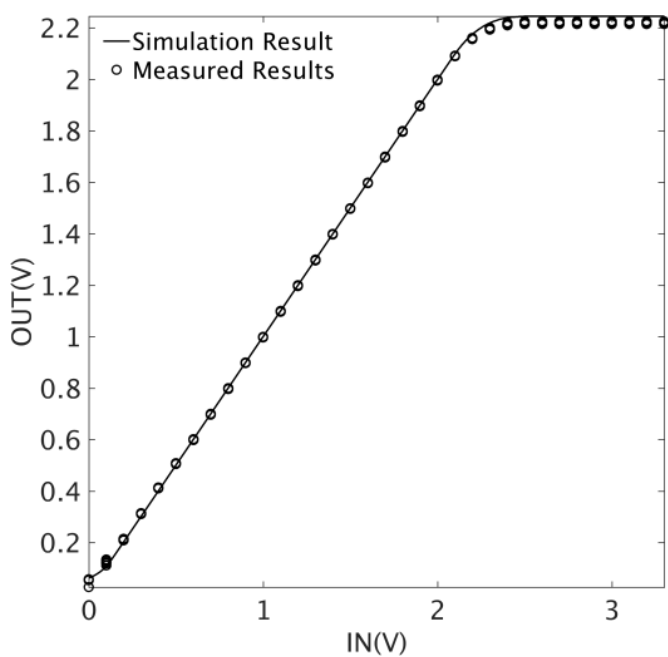

Fig. 7. DC characterization results of the defect-free circuit.

the case of open-drain/source defects have a minor contribution to the DC conditions, in contrast to open-gate defects [23].

In order to model this small amount of DC current, a highvalue resistance model has been proposed in previous work [24]. This approach is, indeed, very successful in DC fault simulations, since it prevents possible convergence problems that can occur in case of the floating nodes. However, choosing the value of this resistance arbitrarily might lead to misleading results in terms of test the selection in defect-oriented methods. Thus, in this work, it is proposed to use a range of possible values (e.g. from $1 \mathrm{G} \Omega$ to $1 \mathrm{~T} \Omega$ ) and to define a region of possible results. The simulation effort can further be simplified by simulating only the largest and the smallest values to define the limits of this region, once it is verified that the results are behaviorally similar.

In spite of the simplicity and the success of this approach in DC modeling, it is not possible to model the complete floating behavior where the current flowing on the affected branch is almost zero. According to the location of the defect, it is, yet, possible to have almost no current flow, as will be 


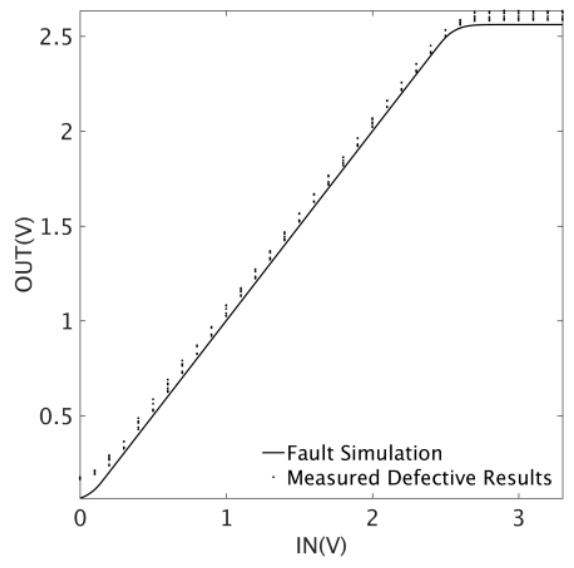

(a)

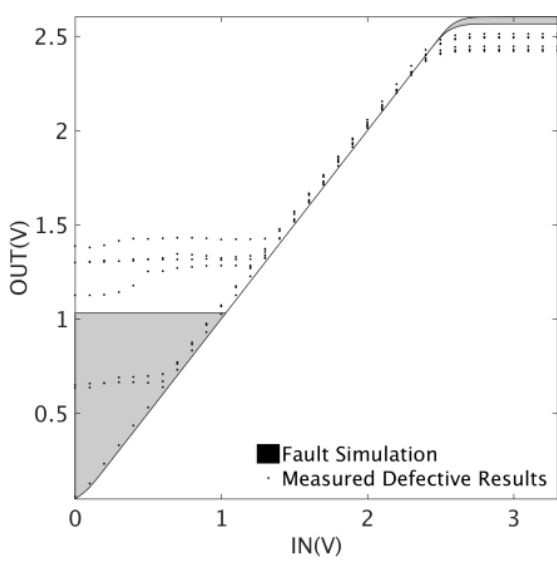

(b)

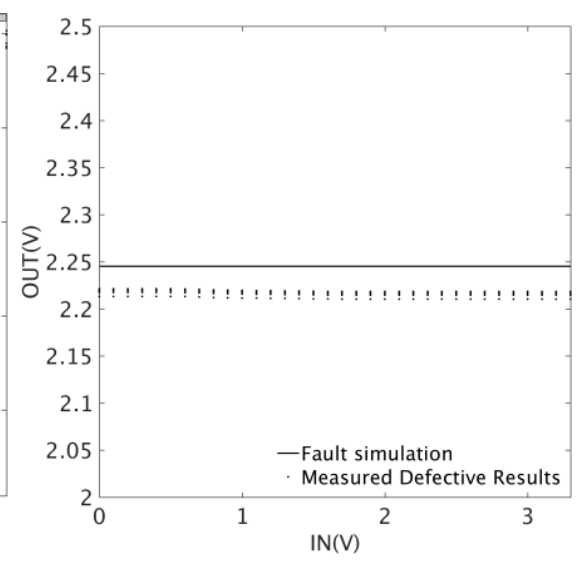

(c)

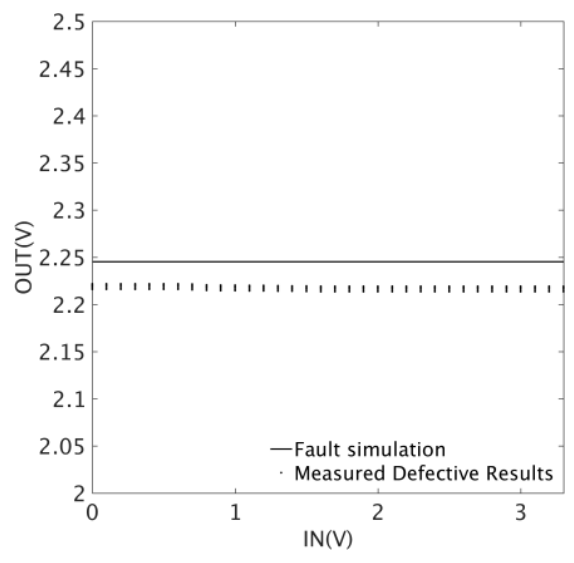

(d)

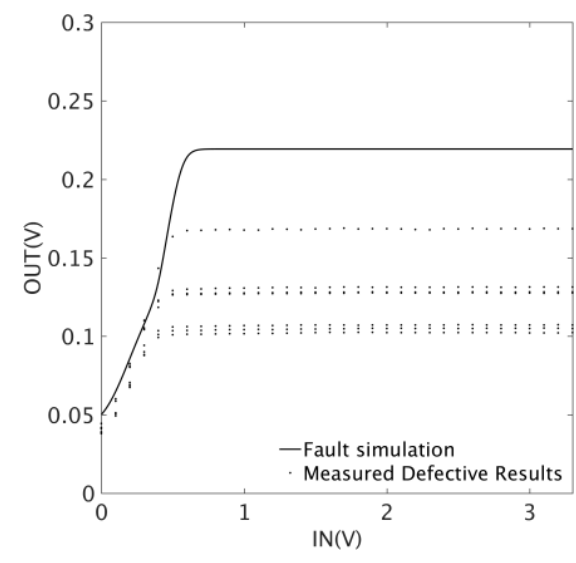

(e)

Fig. 8. DC charaterization results showing the comparison between the simulation results with the subthreshold transistor model and the measurements of 10 samples for (a) defect 1, (b) defect 2, (c) defect 7, (d) defect 8, (e) defect 10

demonstrated by the experimental results. This situation in that case can be modeled by a DC current source with zero amplitude. Moreover, the use of a DC current source instead of a high-value resistor can easily be extended to model also the leakage currents. In most of the cases, however, a complete floating model is not needed, since this kind of defects results in easily detectable responses. Therefore, it might not be worthy to use extensive models in terms of the defect-oriented approach. While the high-value-resistance model is validated by the experimental results, the need for modeling the complete floating behavior will be discussed as well in Section IV.

\section{EXPERIMENTAL RESULTS}

Fig. 5 shows the schematic of the test circuit used to validate the proposed fault models. It is a folded-cascode amplifier circuit with class- $A B$ output stage, which is a common building block in analog and mixed-signal ICs. For measurement purposes, it is configured as a unity-gain buffer by internal negative feedback. Fig. 5, also, indicates the location of each injected open defect in the circuit schematic. Fig. 6 shows the die micrograph of the test chip, fabricated in $0.35 \mu \mathrm{m}$ BCD technology, which includes one defect-free and 10 different intentionally defective versions of the test circuit. All circuits share the same input signal, supply and bias lines, whereas each circuit has an individual output pin in order to prevent possible loading effects that might be introduced by parallelizing measurement methods.

All the open defects are injected by intentionally creating small gaps in metal tracks, except for one of the opens on the gate terminal of the input transistor (i.e. defect 8). Defect 8 is created by removing metal to polysilicon contacts in order to observe the effect of the defect location, as discussed in Section II. Furthermore, due to the presence of one defect-free version of the circuit on the same die, it is possible to analyze the effect of the process variations and to propose a testing approach by comparing the defect-free results with the defective ones (see Section IV).

Fig. 7 shows the DC characterization results of 10 samples of the defect-free circuit in comparison with the simulation results. In order to introduce the least possible loading effects, especially for the defective circuits, the output voltages are measured across the terminals of a true current source configured to conduct zero current.

\section{A. Open-Gate Results}

Fig. 8 shows the fault simulation results using the proposed subthreshold transistor model in comparison with the 


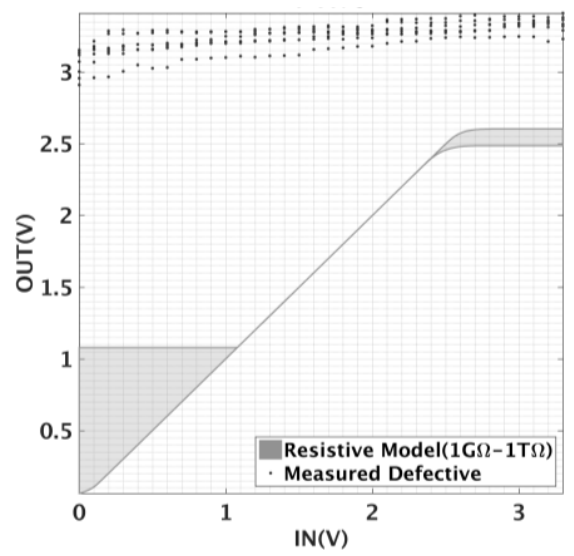

(a)

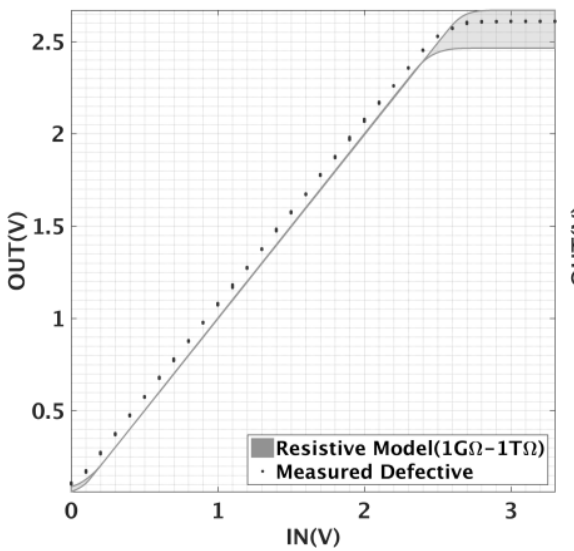

(b)

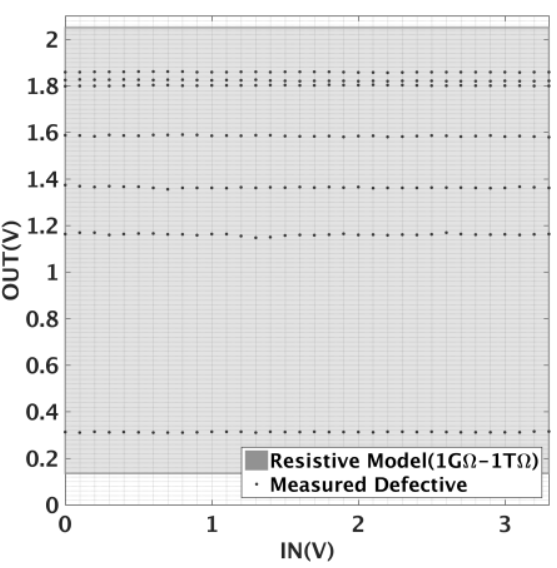

(c)

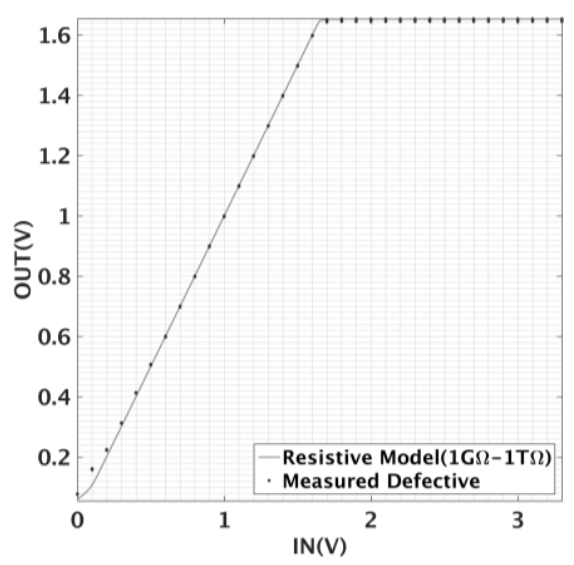

(d)

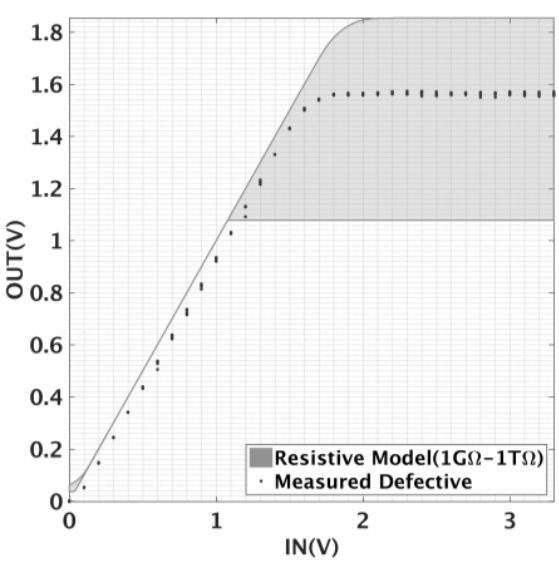

(e)

Fig. 9. DC characterization results showing the comparison between the simulatiom results with the high-value-resistance model and the measurements of 10 samples for (a) defect 3, (b) defect 4, (c) defect 5, (d) defect 6, (e) defect 9.

measurement results of 10 samples for each defective circuit. In general, the agreement between the measurement results and the fault simulation results validates the proposed fault model. It is also shown that for most of the open-gate defects one fault simulation per defect is sufficient to model the defective behavior. However, for the sake of generality, it is required to take the process variations into account, as demonstrated by Fig. 8(b) and (e).

The effect of any random defect parameters such as the initial charge trapped in the gate (i.e. $\mathrm{Q}_{0}$ ) can be analyzed by comparing the measurement results of different samples for each open-gate defect. The uniform behavior of the different samples, particularly in Fig 8(a), (c) and (d), proves that $\mathrm{Q}_{0}$ is not a dominant factor in the open-gate modeling for analog circuits, as predicted in Section II.

It has been discussed that missing metal to polysilicon contacts or breaks in polysilicon are unlikely reasons to create an entire transistor with a floating gate. Nevertheless, an opengate defect on the input transistor is injected in two different ways in the test chip in order to investigate the defect-location dependence of the two overlap capacitances, i.e. $\mathrm{C}_{\mathrm{mpo}}$ and $\mathrm{C}_{\mathrm{pbo}}$. While defect 7 is formed by a small open on a metal track, defect 8 is formed by two missing contacts between the metal and polysilicon layers. As shown in Fig. 8(c) and (d), the agreement between the measurement results for defect 7 and defect 8 proves that $\mathrm{C}_{\mathrm{mpo}}$ and $\mathrm{C}_{\mathrm{pbo}}$ can be neglected independently of the location of the defect for analog transistors.

\section{B. Open-Drain Results}

Fig. 9 shows the fault simulation results using the highvalue-resistance model with the proposed improvement in comparison with the measurement results of 10 samples for each defective circuit. In general, the agreement between the measurement results and the fault simulation results validates the proposed model. Fig. 9(a), however, shows a discrepancy between the fault simulations and the measurements, which exhibit complete floating behavior. The physical reason for this behavior is that defect 3 is an open drain on a p-channel transistor which has the body and the source terminals connected together to the output of the circuit. As a result, the leakage current to the body of the transistor is blocked by the true current source used in the measurements. However, in many practical applications where the output of the circuit is connected to at least a leakage path, the complete floating behavior is not likely to be observed. Furthermore, the circuits with this kind of defects are easily detectable as is clear in Fig. 


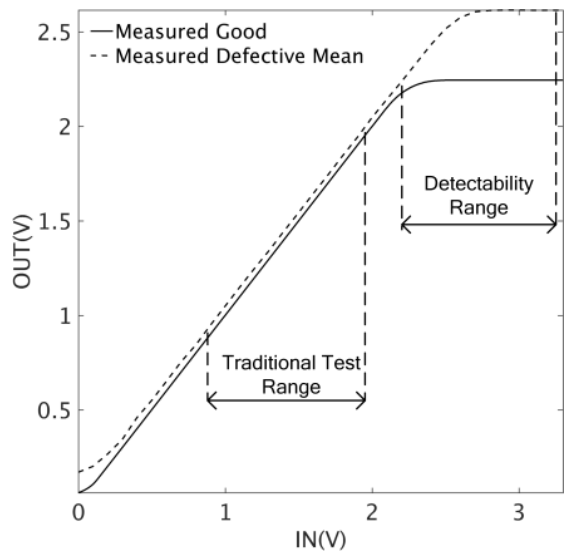

(a)

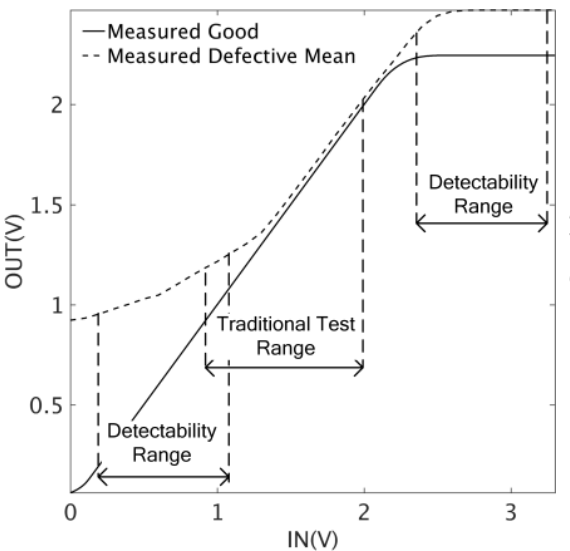

(b)

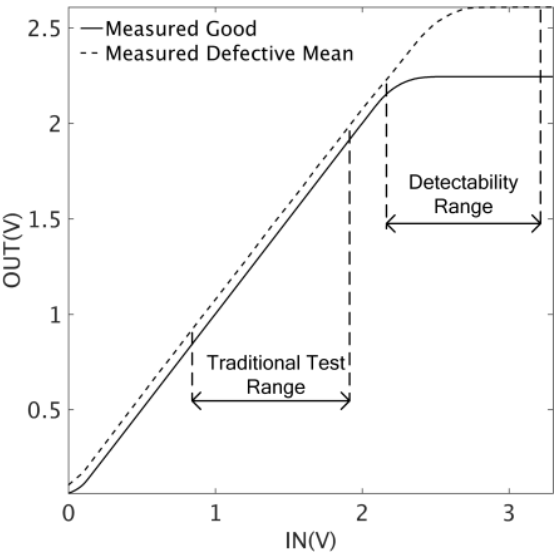

(c)

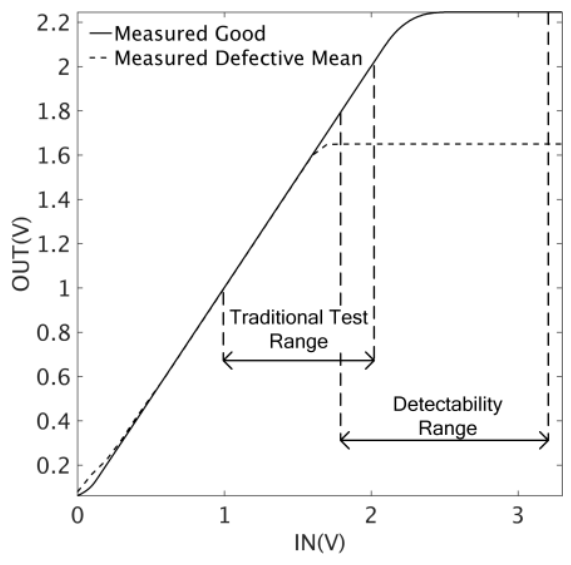

(d)

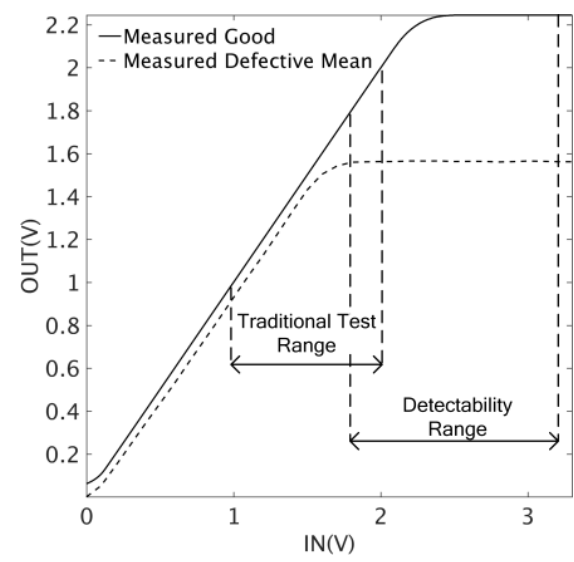

(e)

Fig. 10. Actual detectability and tradional test ranges for (a) defect 1, (b) defect 2, (c) defect 4, (d) defect 6, (e) defect 9.

9(a). Therefore, in terms of the defect-oriented test approach, it is not needed to model this behavior.

The necessity of using a range of high-value resistances rather than one arbitrarily chosen value can be observed, particularly, in Fig 9(b) and (e), whereas one fault simulation is sufficient for defect 6. Furthermore, Fig. 9(c) shows that the process variations should be also taken into account in the fault simulations, although defect 5 is easily detectable.

\section{TESTING APPROACH}

Open defects occurring in signal paths are generally detectable by the traditional DC tests as shown by the measurement results. However, there are also hard-to-detect open defects that are, for example, in the biasing circuitry. The detection of these defects is possible with more complex testing methods in which timing information and frequency responses are used, since most of such open defects result in settling problems. DC tests, on the other hand, are advantageous because of their low cost, widespread applicability and availability. Furthermore, DC test sets are easier to generate in a defect-oriented testing methodology, since the required simulation effort is lower, hence the importance of DC testing.
The use of $\mathrm{I}_{\mathrm{DDQ}}$ testing has been proven to be successful for open-gate defects in digital circuits, since they cause a quiescent current flow, as discussed above too. However, it is not as effective in analog circuits, because the shift in quiescent current caused by any open-gate defect is typically not separable from the one caused by process variations.

While traditional DC tests, which are driven by the performance specifications, are focused on the functional regions of the circuits, most of the open defects become detectable with the input values near the supply voltages, as shown by Fig. 10. This suggests that DC tests that force transistors to operate outside their designed operation region are favorable to detect open defects. Therefore, the addition of measurement points from the common detectability ranges shown in Fig. 10 to the specification-based DC tests can increase the fault coverage at very low cost. This approach has two important advantages. First, the increase in test time introduced to the specification-based tests is minimal. Besides, it is a predictive defect-oriented testing method in which excessive fault simulations are not required.

In order to verify the proposed predictive method, DC fault simulations with the validated fault models was carried out for the experimental test circuit. There are 30 possible open defects 
out of which 16 defects are easily detectable by the traditional DC tests. The proposed approach, which involves the addition of the measurement points from the predicted detectability range, ensures the detection of the remaining 13 hard-to-detect open defects. In other words, the proposed predictive approach improves the fault coverage for open defects from 53,3\% up to $96,6 \%$ at the expense of the minimal increase in terms of the test cost.

Although the proposed approach is explained by a subset of the open defects in a specific experimental circuit, it can be extended to many analog circuits. This is mainly because the effect of all open defects, which is a decrease in the quiescent current in one or more branches, is behaviorally identical for analog circuits. The lack of a proper bias current in analog branches can be made observable by changing the operation region of the transistors, since this change is not possible in case of open defects. In other words, DC operating points and a small subthreshold or leakage current in the branches that are affected by open defects are sustained independently of the neighboring nodes. In this paper, the use of the available input signals is proposed to expose the discussed effect. However, supply voltages, in the same manner, can also be used in order to force transistors to operate in different regions.

\section{CONCLUSIONS}

Open defects in analog circuits have been analyzed in terms of their electrical properties. A new DC open-gate model, in which the common characteristics of analog transistors are considered, has been proposed. The model is based on voltagedependent voltage sources, which keep the transistor in the subthreshold region as has been concluded from the in-depth analysis. The measurement results of an experimental test chip fabricated in $0.35 \mu \mathrm{m}$ BCD technology have been presented to validate the proposed model and high-value-resistance model for open-drain/source defects. Finally, a predictive defectoriented testing approach has been proposed, which addresses DC testability regions for open defects in analog circuits. The testing approach is based on forcing transistors to operate outside their designed regions to make open defects observable in DC test conditions.

\section{ACKNOWLEDGMENT}

This research has been carried out within the framework of the SAFE-IC project funded by the IWT (code: 120642).

\section{REFERENCES}

[1] W. Dobbelaere, R. Vanhooren, A. Coyette, and G. Gielen, "Analog fault coverage improvement using defect-specific masking," VOICE conference, 2014.

[2] P. Maxwell, I. Hartanto, and L. Bentz, "Comparing functional and structural tests," International Test Conference (ITC), pp. 400-407, 2000.

[3] G. Gielen, W. Dobbelaere, R. Vanhooren, A. Coyette, and B. Esen, "Design and test of analog circuits towards sub-ppm level." International Test Conference (ITC), pp. 1-2, 2014.
[4] L. Abdallah, H.G. Stratigopoulos, S. Mir, and J. Altet, "Defect-oriented non-intrusive RF test using on-chip temperature sensors," VLSI Test Symposium (VTS), pp. 1-6, 2013.

[5] A. Coyette, B. Esen, R. Vanhooren, W. Dobbelaere, and G. Gielen, "Automatic generation of lightweight controllability and observability structures for analog circuits," Synthesis, Modeling, Analysis and Simulation Methods and Applications to Circuit Design (SMACD), pp. $1-4,2015$

[6] S. Sunter, and N. Nagi, "Test metrics for analog parametric faults," VLSI Test Symposium, pp. 226-234, 1999.

[7] B. Kruseman, B. Tasić, C. Hora, J. Dohmen, H. Hashempour, M. van Beurden, and Y. Xing, "Defect oriented testing for analog/mixed-signal devices," International Test Conference (ITC), pp. 1-10, 2011.

[8] H. Banba, H. Shiga, A. Umezawa, T. Miyaba, T. Tanzawa, S. Atsumi, and K. Sakui, "A CMOS bandgap reference circuit with sub-1-V operation," IEEE Journal of Solid-State Circuits, vol. 34, no. 5 pp. 670674, 1999.

[9] A. M. Pappu, X. Zhang, A. V. Harrison, and A. B. Apsel, "Processinvariant current source design: Methodology and examples," IEEE Journal of Solid-State Circuits, vol. 42, no. 10, pp. 2293-2302, 2007.

[10] S. Borkar, T. Karnik, S. Narendra, J. Tschanz, A. Keshavarzi, and V. De, "Parameter variations and impact on circuits and microarchitecture," Design Automation Conference, pp. 338-342, 2003.

[11] M. Soma,. "Analog fault models: Back to the future?," Internetional Test Conference (ITC), 2014.

[12] M. Renovell, and G. Cambon, "Electrical analysis and modeling of floating-gate fault," Transactions on CAD of Integ. Circuits and Systems, vol. 11, no. 11, pp. 1450-1458, Nov. 1992.

[13] A. Ivanov, S, Rafiq, M. Renovell, F. Azaïs, and Y. Bertrand, "On the detectability of CMOS floating gate transistor faults," Transactions on CAD of Integ. Circuits and Systems, vol. 20, no. 1, pp. 116-128, 2001.

[14] V.H. Champac, J.A. Rubio, and J. Figueras, "Electrical model of the floating gate defect in CMOS Ics: Implication on IDDQ testing," Trans. Computer-Aided Design, vol. 13, pp. 359-369, Mar. 1994.

[15] A.M. Brosa, and J. Figueras, "Characterization of floating gate defects in analog cells," Journal of Electronic Testing, vol.14, no. 1-2, pp. 23-31, 1999.

[16] M. Pronath, H. Graeb, and K. Antreich, "A test design method for floating gate defects (FGD) in analog integrated circuits," Design, Automation and Test in Europe(DATE), pp. 78, 2002.

[17] A. Kolodny, S. T. K. Nieh, B. Eitan, and J. Shappir, "Analysis and modeling of floating gate EEPROM cells," IEEE Trans. Electron Devices, vol. ED-33, pp. 835-844, June 1986.

[18] S. Chung, C.-M. Yih, S. S.Wu, H. H. Chen, and G. Hong, "A spicecompatible Flash EEPROM model feasible for transient and program/erase cycling endurance simulation," IEDM Tech. Dig., pp. 179-182, 1999.

[19] L. C. Liong and P. Liu, "A theoretical model for the current-voltage characteristics of a floating-gate EEPROM cell," Transactions Electron Devices, vol. 40, pp. 146-151, Jan. 1993.

[20] A.F. Mondragón-Torres, M.C. Schneider, and E. Sánchez-Sinencio, "Extraction of electrical parameters of floating gate devices for circuit analysis, simulation, and design," MWSCAS, vol. 1, pp. I-311, 2002.

[21] L. Larcher, P. Pavan, S. Pietri, L. Albani, and A. Marmiroli, "A new compact DC model of floating gate memory cells without capacitive coupling coefficients," Transactions Electron Devices, vol. 49, no. 2, pp. 301-307, 2002.

[22] Y. Tsividis, Operation and modeling of the MOS transistor, 2rd ed., Oxford University Press, pp.385-405, 1999.

[23] E. Acar, and S. Ozev, "Defect-based RF testing using a new catastrophic fault model," International Test Conference (ITC), pp. 9-17, 2005.

[24] T. Olbrich, J. Perez, I. Grout, A. Richardson, and C. Ferrer, "Defectoriented vs schematic-level based fault simulation for mixed-signal ICs," International Test Conference (ITC), pp. 511-520, 1996. 